\title{
UTILIZATION OF ANCILLARY DATA SETS FOR SMAP ALGORITHM DEVELOPMENT AND PRODUCT GENERATION
}

\author{
P. O’Neill \\ Hydrological Sciences Branch / Code 614.3 \\ NASA Goddard Space Flight Center, Greenbelt, MD 20771 USA \\ tel: 1-301-614-5773, fax: 1-301-614-5808, Peggy.E.ONeill@nasa.gov \\ E. Podest, E. Njoku \\ Jet Propulsion Laboratory, California Institute of Technology \\ Pasadena, CA 91109 USA
}

\section{INTRODUCTION}

The Soil Moisture Active Passive (SMAP) mission is one of the first Earth observation satellites being developed by NASA in response to the National Research Council's Decadal Survey, Earth Science and Applications from Space: National Imperatives for the Next Decade and Beyond [1]. Scheduled to launch late in 2014, SMAP will provide high resolution and frequent revisit global mapping of soil moisture and freeze/thaw state, utilizing enhanced Radio Frequency Interference (RFI) mitigation approaches to collect new measurements of the hydrological condition of the Earth's surface. The SMAP instrument design incorporates an L-band radar (3 km) and an L band radiometer (40 km) sharing a single 6-meter rotating mesh antenna to provide measurements of soil moisture and landscape freeze/thaw state [2]. These observations will (1) improve our understanding of linkages between the Earth's water, energy, and carbon cycles, (2) benefit many application areas including numerical weather and climate prediction, flood and drought monitoring, agricultural productivity, human health, and national security, (3) help to address priority questions on climate change, and (4) potentially provide continuity with brightness temperature and soil moisture measurements from ESA's SMOS (Soil Moisture Ocean Salinity) and NASA’s Aquarius missions. In the SMAP prelaunch time frame, baseline algorithms are being developed for generating (1) soil moisture products both from radiometer measurements on a $36 \mathrm{~km}$ grid and from combined radar/radiometer measurements on a $9 \mathrm{~km}$ grid, and (2) freeze/thaw products from radar measurements on a $3 \mathrm{~km}$ grid. These retrieval algorithms need a variety of global ancillary data, both static and dynamic, to run the retrieval models, constrain the retrievals, and provide flags for indicating retrieval quality. The choice of which ancillary dataset to use 
for a particular SMAP product will be based on a number of factors, including its availability and ease of use, its inherent error and resulting impact on the overall soil moisture or freeze/thaw retrieval accuracy, and its compatibility with similar choices made by the SMOS mission. All decisions regarding SMAP ancillary data sources will be fully documented by the SMAP Project and made available to the user community.

\section{SMAP ANCILLARY DATA}

Ancillary data needed by the SMAP mission fall into two categories -- static ancillary data are data which do not change during the mission while dynamic ancillary data require periodic updates in time frames ranging from seasonally to daily. Static data include parameters such as permanent masks (land/water/forest/urban/mountain), the grid cell average elevation and slope from a DEM, permanent open water fraction, and soils information (primarily sand and clay fraction). The SMAP Project plans to create a master file of all of the static ancillary data resampled to the same grids as the output products, and this master file would be available to any algorithm or end user who needs it. The dynamic ancillary data include land cover, roughness, vegetation parameters, and effective soil temperatures. Measurements from the SMAP radar will be used to provide information primarily on transient water and frozen ground.

Ancillary data will also be employed to set flags which help to determine either specific aspects of the processing (such as corrections for transient water) or the quality of the retrieved soil moisture (e.g. precipitation flag). Basically, these flags would provide information as to whether the ground is frozen, snow-covered, or flooded, or whether it is actively precipitating at the time of the satellite overpass. Other flags will indicate whether masks for steeply sloped topography, or for urban, heavily forested, or permanent snow/ice areas are in effect.

\section{ANCILLARY DATA SELECTION PROCESS}

A rigorous ancillary dataset selection process has been undertaken in order to consider all datasets available for each parameter and to ensure consistency in the datasets used throughout the different retrieval algorithms for the different SMAP baseline products. Consistency with SMOS products is also a consideration. Key factors considered in the ancillary data selection are latency, spatial resolution, temporal resolution, global coverage, accessibility/ease of use, and quality checks/internal error. Once a dataset has been selected as the primary source for a given parameter, it is transferred onto the SMAP 
testbed for regridding in a consistent manner (used by other datasets) to the resolution needed by the retrieval algorithms. Flags and masks are also generated for the appropriate parameters. Documentation of the selection rationale for a given dataset will include specification of a primary and secondary dataset as well as anticipated new and improved datasets that will potentially replace the selected one by the time SMAP is in orbit.

An example of a selected ancillary dataset is shown in Figure 1. SMAP retrieval algorithms require information about soil texture, specifically sand and clay fraction. A global dataset was assembled from an optimized combination of the FAO (Food \& Agriculture Organization), HWSD (Harmonized World Soil Database), STATSGO (State Soil Geographic_US), NSDC (National Soil Database Canada), and ASRIS (Australian Soil Resources Information System) soil databases, using the best available source for a given region [3]. Similar analyses have been conducted for selection of a permanent open water fraction dataset (MODIS44W global static product at $250 \mathrm{~m}$ resolution [4]) and land surface class (MODIS IGPB global product at $250 \mathrm{~m}$ resolution). Selection of other datasets is currently in progress for example, preliminary analysis of ECMWF (European Centre for Medium-Range Weather Forecasts) and GMAO MERRA (Goddard Global Modeling \& Assimilation Office Modern Era RetrospectiveAnalysis For Research and Applications) soil and air temperature products indicates that both sources may meet the $2{ }^{\circ} \mathrm{C}$ accuracy desired for soil moisture retrieval [5].

\section{SUMMARY}

Algorithms being developed for the SMAP mission require a variety of both static and ancillary data. The selection of the most appropriate source for each ancillary data parameter is driven by a number of considerations, including accuracy, latency, availability, and consistency across all SMAP products and with SMOS. It is anticipated that initial selection of all ancillary datasets, which are needed for ongoing algorithm development activities on the SMAP algorithm testbed at JPL, will be completed within the year. These datasets will be updated as new or improved sources become available, and all selections and changes will be documented for the benefit of the user community. Wise choices in ancillary data will help to enable SMAP to provide new global measurements of soil moisture and freeze/thaw state at the targeted accuracy necessary to tackle hydrologically-relevant societal issues. 


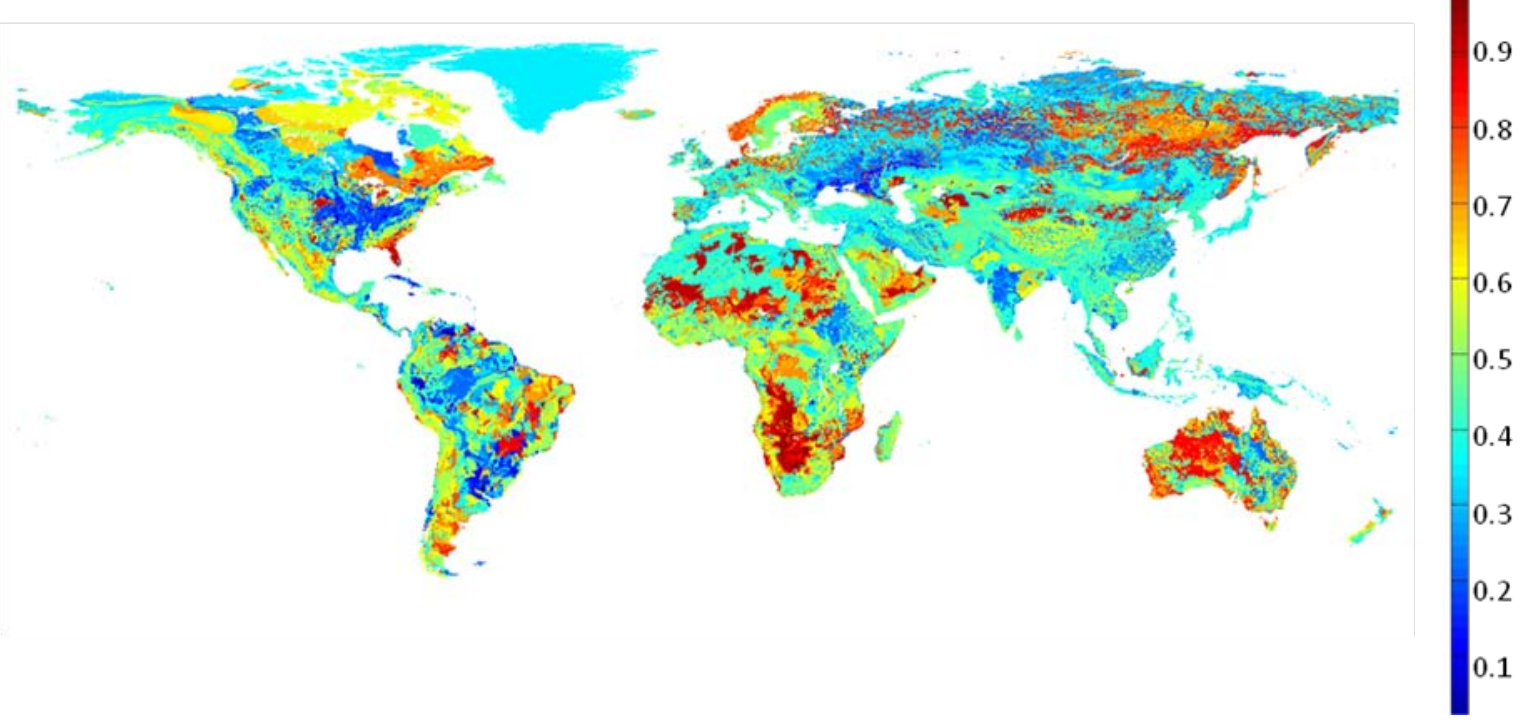

Figure 1. Global sand fraction at 0.01 degree resolution based on a composite of FAO, HWSD, STATSGO, NSDC, and ASRIS datasets using best available source for a given region.

\section{REFERENCES}

[1] National Research Council, "Earth Science and Applications from Space: National Imperatives for the Next Decade and Beyond,” pp. 400, 2007.

[2] Entekhabi, D., E, Njoku, P. O’Neill, K. Kellogg, plus 19 others, "The Soil Moisture Active Passive (SMAP) Mission,” Proceedings of the IEEE, Vol. 98, No. 5, May, 2010.

[3] Das, N. and P. O'Neill, "Selection of Soil Attributes Datasets for the SMAP Mission," SMAP Science Document \#xz, Draft 1.1, Jet Propulsion Laboratory, December, 2010.

[4] Steven Chan, "Selection of a Water-Land Fraction Dataset for the SMAP Mission," SMAP Science Document \#xx, Draft 1, Jet Propulsion Laboratory, November, 2010.

[5] Holmes, T., T. Jackson, R. Reichle, and J. Basara, "Validation of soil temperature datasets against ground measurements in Oklahoma," in preparation, 2011. 